\title{
Extreme Thrombocytosis Resulting from Minimally Invasive Foot Surgery with Forefoot Necrosis: A Rare Case Report
}

\author{
${ }^{1}$ Department of Orthopaedics Surgery, Gregorio Marañón University Hospital, Spain \\ ${ }^{2}$ Department of Anaesthesiology, Gregorio Marañón University Hospital, Spain \\ ${ }^{3}$ Department of Plastic Surgery, Gregorio Marañón University Hospital, Spain
}

Cuervas-Mons Manuel ${ }^{1}$, Álvarez Baena Lucía ${ }^{2}$, García Ruano Angela ${ }^{3}$ and Arnal Burró Javier

*Corresponding author: Manuel Cuervas-Mons Cantón, Department of Orthopaedics Surgery, Gregorio

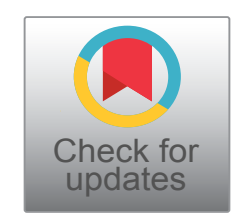
Marañón University Hospital, c/Dr Esquerdo 46, 28007 Madrid, Spain, Tel: 0034-915868426

\begin{abstract}
Essential thrombocythemia is a chronic myeloproliferative syndrome characterised by excessive proliferation of platelets and megakaryocytes, with a relatively favourable course. We present a case of extreme thrombocytosis resulting from minimally invasive foot surgery with forefoot necrosis. A 72-year-old female patient presented with hallux valgus and metatarsalgia. A platelet count of $560,000 /$ $\mu \mathrm{L}$ was detected as an unexpected finding. Minimally invasive surgery was performed, and in the first post-surgical week, skin lesions appeared on the forefoot. Three weeks later, the patient presented complete necrosis of the first toe and phlegmonosis of the forefoot. On admission, a platelet count of $>1,000,000 / \mu \mathrm{l}$ was observed. Multidisciplinary treatments were conducted. Initially, ASA $100 \mathrm{mg} / 24 \mathrm{~h}$ and hydroxyurea $500 \mathrm{mg} / 24 \mathrm{~h}$ were administrated, and clinical and analytical improvements were achieved. Two weeks later, transmetatarsal amputation and cutaneous advance flap were operated as definitive treatment. Six months later the treatment with hydroxyurea was maintained with a platelet count of $290,000 / \mu \mathrm{l}$. In conclusion, mild thrombocythemia is considered as an unexpected finding of uncertain significance and does not contraindicate surgical treatment. Patients with mild thrombocytosis in the preoperative stage could benefit from prophylactic ASA $100 \mathrm{mg} / 24 \mathrm{~h}$ until the surgical treatment is completed, given the risk/benefit balance.
\end{abstract}

\section{Keywords}

Essential thrombocythemia, Foot, Necrosis, Minimally invasive surgery, MIS, Reactive Thrombocytosis, Extreme thrombocytosis

\section{Introduction}

Essential thrombocythemia (ET) is a chronic myeloproliferative syndrome characterised by excessive proliferation of platelets and megakaryocytes, with a relatively favourable course [1]. The aetiology of the thrombocythemia can be clonal (due to a genetic alteration) or reactive (secondary to an inflammatory process) [2]. The cardinal symptoms are due to thromboembolism and microvascular damage caused by an increased amount of platelets. Thrombocytosis is detected fortuitously in routine examinations in most patients, whereas it produces clinically relevant alterations only on rare occasions. We present a case of extreme thrombocytosis resulting from minimally invasive foot surgery with forefoot necrosis.

\section{Case Report}

A 72-year-old female patient was diagnosed with hallux valgus and metatarsalgia. The patient had no medical allergies or significant personal, medical or surgical histories. After 12 months of orthopodological treatment, the patient continued with pain, and surgical treatment was performed. A complete preoperative study was carried out and classified as ASA II (American Society of Anesthesiologist physical status classification). A platelet count of $560,000 / \mu \mathrm{L}$ was detected as an unexpected finding. Her primary care physician recommended the patient for monitoring platelet, but the patient did not give it importance. Minimally in- 

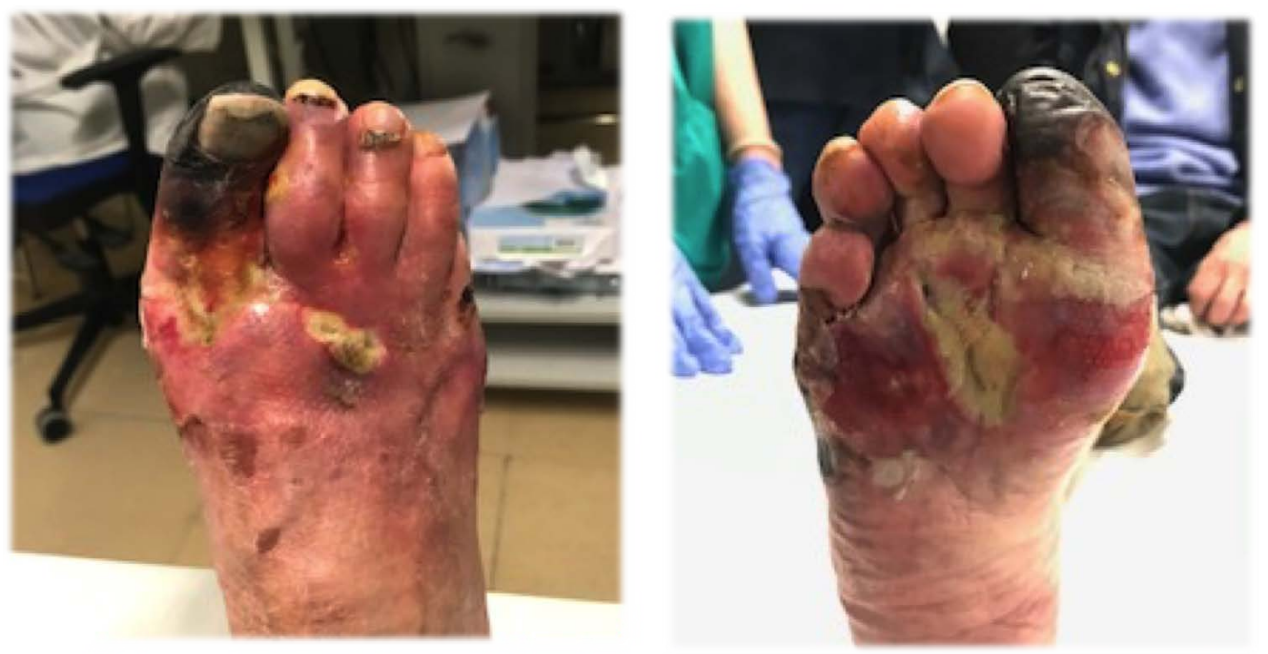

Figure 1: Forefoot necrosis and phlegmonosis.
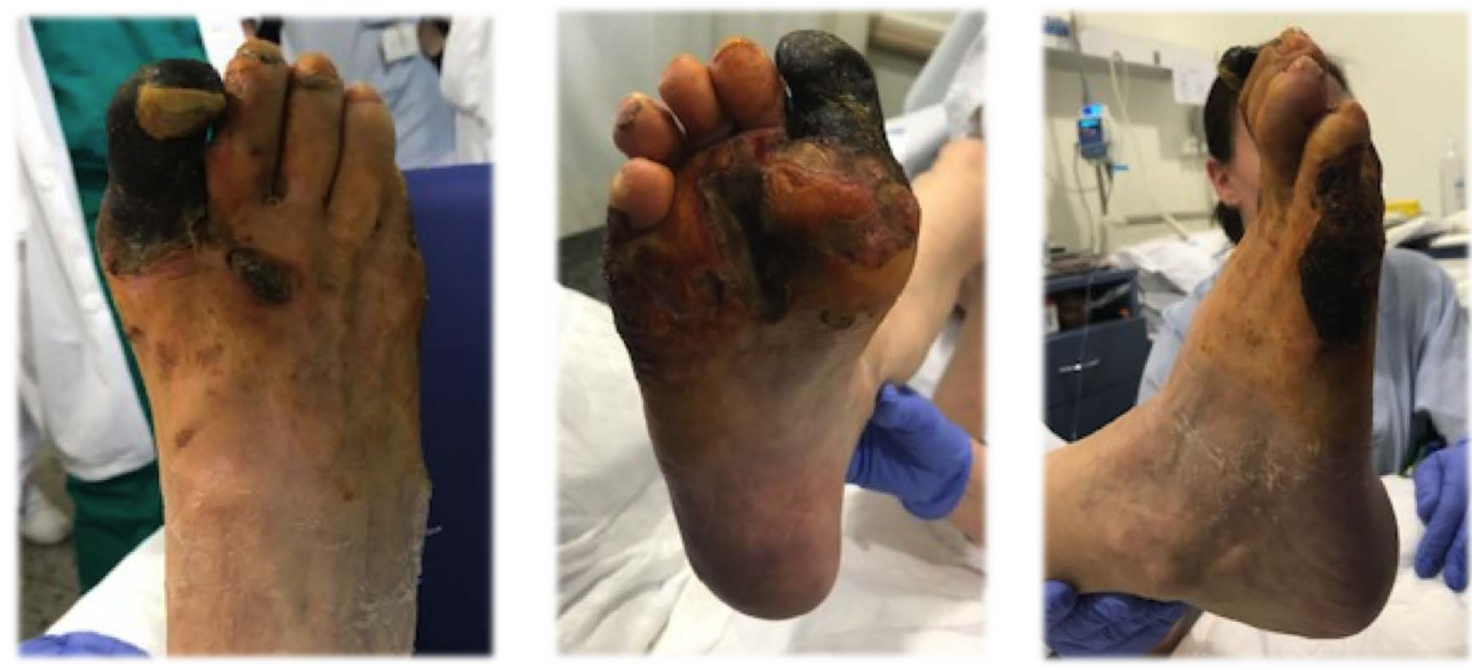

Figure 2: Delimited necrotic zone.

vasive surgery (MIS) was performed at a local hospital without incidences according to the surgical records, performing: first metatarsal distal exostectomy, Akin osteotomy (closing wedge osteotomy in the proximal phalanx of the first toe) and distal metatarsal minimal invasive osteotomies (DMMO) in the second and third metatarsal. The patient reported that in the first post-surgical week, skin lesions appeared on the forefoot, treated with periodic cures every 48 hours with povidone-iodine and saline, until the third post-surgical week when the patient rushed to the emergency department of our centre due to poor analgesic control. Upon arrival, the patient presented with complete necrosis of the first toe, necrotic plaque on the lateral side of the foot, dorsal and plantar phlegmonosis of the forefoot, and hindfoot venous congestion signs (Figure 1). Hospital admission and epidural catheter placement for analgesic control were conducted. On admission, complete blood count shown: haemoglobin $13.8 \mathrm{~g} / \mathrm{dl}$, leucocyte count $18.1 \times 10 \mathrm{E} 3 / \mu \mathrm{l}$, platelet count of $>1 \times$ $10 \mathrm{E} 6 / \mu \mathrm{l}$, and $\mathrm{C}$ reactive protein $3.9 \mathrm{mg} / \mathrm{dL}$. A peripheral blood smear was performed, showing normal platelet morphology. The patient was initially diagnosed with extreme thrombocytosis and possible reactive thrombocytosis. Multidisciplinary treatments were performed with Hematology, and Surgical Services (Vascular, Plastic and Orthopaedics). A complete haematological study was performed, and positive JAK2V617F mutation was detected. The patient was diagnosed with essential thrombocytosis with a reactive component. Initial treatment with acetylsalicylic acid (ASA) $100 \mathrm{mg} / 24 \mathrm{~h}$ and hydroxyurea $500 \mathrm{mg} / 24 \mathrm{~h}$ was given, and clinical and analytical improvements were achieved. The necrotic zone was delimited to the first toe, the lateral side of the forefoot and plantar region of central metatarsals (Figure 2). Two weeks later, transmetatarsal amputation and cutaneous advance flap were performed as definitive treatment (Figure 3). During the postoperative period, there were no complications, with good skin evolution (Figure 4). Six months later, the treatment with hydroxyurea was maintained and had a platelet count of $290,000 / \mu l$. 


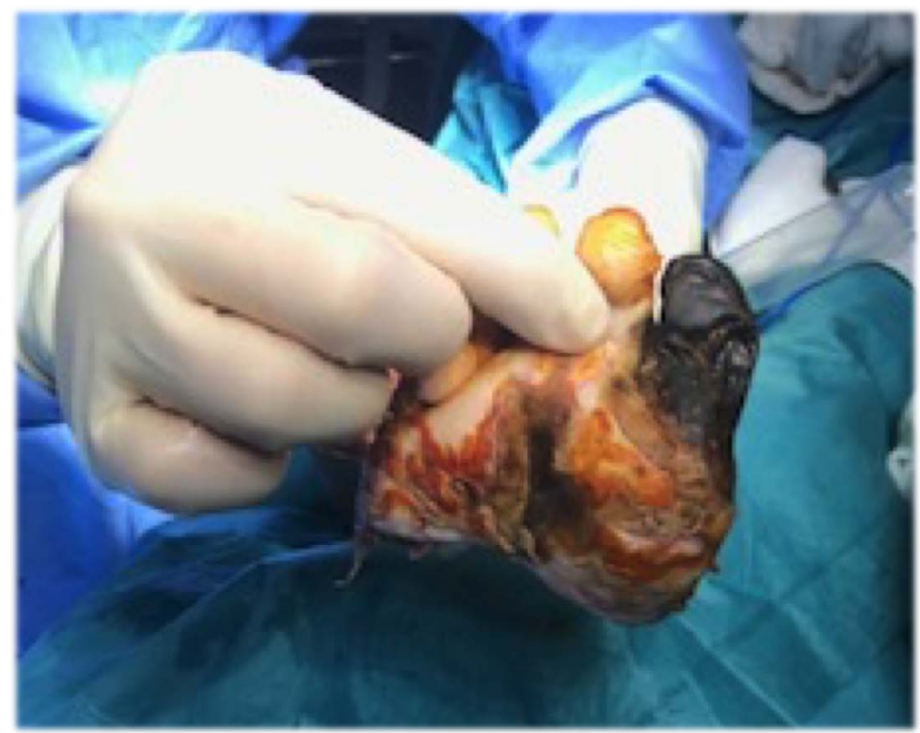

Figure 3: Transmetatarsal amputation.
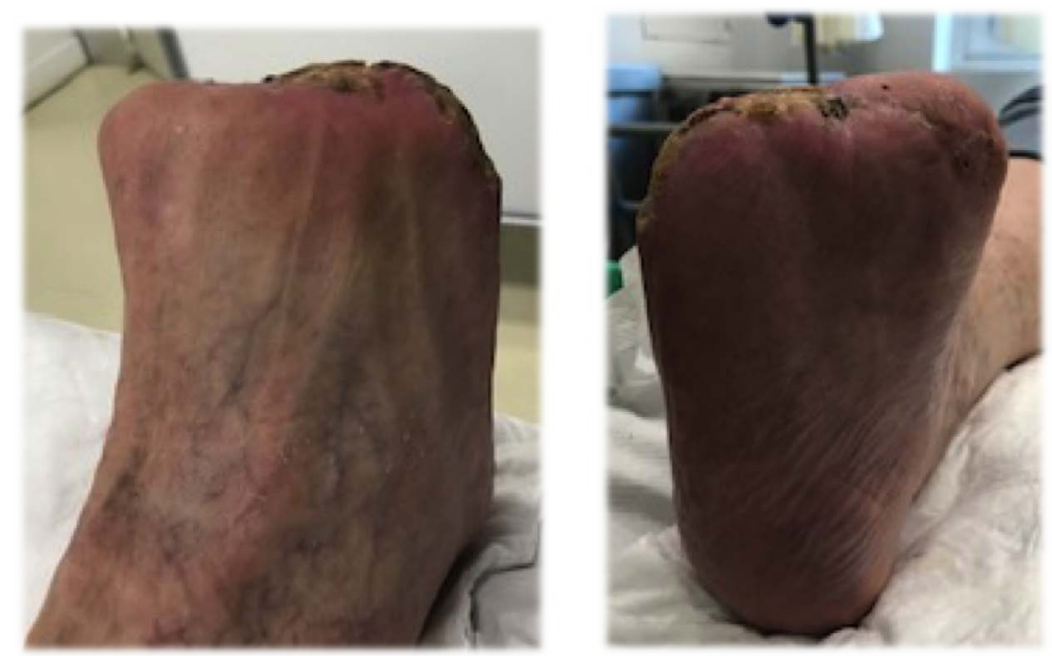

Figure 4: Amputation stump.

\section{Discussion}

Thrombocytosis is an accidental analytical finding on most occasions, and possible methodological errors in the interpretation of the test need initially to be excluded. For the diagnosis of this disease, according to the criteria of the Polycythemia Vera Study Group (PVSG) revised by WHO in 2008, a platelet count of more than $600,000 / \mu \mathrm{l}$ must be observed on two occasions, separated by at least one month (to eliminate a cause of transitory elevation of the platelet) [3,4]. Despite the standard diagnosis criteria are frequently used, they have been recently updated, and a platelet count of more than $450,000 / \mu l$ is now considered as diagnostic criteria for many authors [5].

Elevation in the platelet count can result from physiologic or pathologic mechanisms. Essential thrombocytosis (ET) must be differentiated from secondary thrombocytosis. Secondary thrombocytosis can be caused by many conditions, with the most common being infec- tions, iron deficiency, alcohol consumption, and abundant tissue damage in surgery. Accordingly, secondary thrombocytosis should be the first consideration for a patient like the current case, and its management should be focused on the discovery and treatment of the underlying cause. Extreme thrombocytosis, defined as a platelet count greater than or equal to $1 \times 10 \mathrm{E} 6 /$ $\mu \mathrm{l}$, is rarely seen in general practice, and there are limited data on the aetiology of this abnormality [6]. In published reviews, it is considered that platelet numbers $>$ $1,000,000$ have a reactive cause in $>80 \%$ of cases, but $<4 \%$ of patients with presenting symptoms are related to or attributable to thrombocytosis [7], and there is no vaso-occlusive phenomenon described to date that has required amputation. Reactive thrombocytosis (RT) is an elevated platelet count that develops secondary to another disorder, and the definite diagnosisis based on spontaneous normalisation of platelet count after treatment of the underlying cause. In this patient, an increased $C$ reactive protein and leucocytosis were ob- 
served, associated with extreme thrombocytosis. The tissue damage secondary to surgical procedures can be the underlying cause of $\mathrm{RT}$, but the presence of a potential cause of reactive thrombocytosis does not rule out a concomitant clonal process, especially in persistent thrombocytosis [8].

Available evidence about the risk-benefit ratio of antiplatelet therapy in adults with ET is highly uncertain [9]. The use of ASA at a dose of $100 \mathrm{mg}$ effectively suppresses the production of thromboxane $A 2$, being very effective in controlling ischemic symptoms and therefore plays a fundamental role in ET treatment [10]. The onset of action of ASA is rapid (one hour) and lasts 7-10 days, without significantly increasing the risk of intraoperative bleeding, and its withdrawal is currently not recommended in orthopaedic surgeries [11]. Low-dose ASA treatment (40-325 mg/day) is the cornerstone in the treatment of ET [2], but it is not recommended in the RT [8]. In the treatment of ET, it is used in patients with any degree of risk and reduces the relative risk of thrombosis by $26 \%$ [2]. Nevertheless, RT is felt to be self-limited with little excess associated thrombotic risk and no antiplatelet therapy is recommended, even for extreme thrombocytosis [8].

In many cases, the thrombocytosis is found in the preoperative period as an accidental analytical finding, and differential diagnosis between ET and RT must be performed. In the meanwhile, if the patient needs the surgical procedure, and the underlying cause is still unclear, based on our experience, prophylactic ASA 100 $\mathrm{mg} / 24 \mathrm{~h}$ could be justified until the surgical treatmentis completed. Although further studies are needed, the ASA treatment reduces the relative risk of thrombosis [2] and its withdrawal is currently not recommended in orthopaedic surgeries [8], so given the risk/benefit balance it could be beneficial in this cases.

Mild thrombocythemia is considered as an unexpected finding of uncertain significance and does not contraindicate surgical treatment. Further studies are needed, but from our experience with this patient, we believe that patients with mild thrombocytosis in the preoperative evaluation could benefit from prophylactic ASA $100 \mathrm{mg} / 24 \mathrm{~h}$ until the surgical treatment is completed, given the risk/benefit balance.

\section{References}

1. M Imbert, JW Vardiman (2001) Essential Thrombocythemia. In: Steven H Swerdlow, Elias Campo, Nancy Lee Harris, Elaine S Jaffe, Stefano A Pileri, et al. The WHO Classification of Tumor of Hematopoietic and Lymphoid Tissues. IARC Press, 39-41.

2. Álvarez JF, Bedoya-Trujillo N, Saldaña J (2018) A clinical approach to thrombocytosis, a review of the literature. Salutem Scientia Spiritus 4: 41-48.

3. Murphy S, Peterson P, lland H, Laszlo J (1997) Experience of Polycythemia Vera Study Group with essential thrombocythemia: A final report on diagnostic criteria, survival and leukemic transition by treatment. Sem Hemat 34: 29-39.

4. Brière JB (2007) Essential thrombocythemia. Orphanet $\mathrm{J}$ Rare Dis 2: 3 .

5. Ashorobi D, Gohari P (2019) Essential Thrombocytosis (Essential Thrombocythemia, ET). In: StatPearls. StatPearls Publishing, Treasure Island (FL).

6. Wiwanitkit $\mathrm{V}$ (2006) Extreme thrombocytosis: What are the etiologies? Clin Appl Thromb Hemost 12: 85-87.

7. Buss DH, Cashell AW, O'Connor ML, Richards F 2nd, Case LD (1994) Occurrence, etiology and clinical significance of extreme thormbocytosis: A Study of 280 cases. Am J Med 96: 247-253.

8. Bleeker JS, Hogan WJ (2011) Thrombocytosis: Diagnostic evaluation, thrombotic risk stratification, and risk-based management strategies. Thrombosis 2011: 536062.

9. Chu DK, Hillis CM, Leong DP, Anand SS, Siegal DM (2017) Benefits and risks of antithrombotic therapy in essential thrombocythemia: A systematic review. Ann Intern Med 167: $170-180$.

10. R Hoffman (2000) Prymary Thrombocythemia. In: Hoffman, Hematology: Basic Principles and Practice. Churchill-Livingstone.

11. Kiberd MB, Hall RI (2015) Aspirin in the perioperative period: A review of the recent literature. Curr Opin Anaesthesiol 28: 349-355. 